\title{
Relationship of serum calprotectin, angiopoietin-1, and angiopoietin-2 levels with coronary collateral circulation in patients with stable coronary artery disease
}

\author{
Vahit Demir ${ }^{1}$, Hüseyin Ede, Müjgan Ercan², Yasar Turan¹, Siho Hidayet ${ }^{3}$, Nihan Inandiklioglu4 ${ }^{4}$ Ali Rıza Erbay \\ 1 Department of Cardiology, Medical Faculty, Bozok University, Yozgat, Turkey \\ 2 Department of Biochemistry, Medical Faculty, Harran University, Sanlı Urfa, Turkey \\ 3 Department of Cardiology, Medical Faculty, Inonu University, Malatya, Turkey \\ 4 Department of Medical Biology and Genetics, Medical Faculty, Bozok University, Yozgat, Turkey
}

\section{KEY WORDS}

angiopoietin-1, angiopoietin-2, coronary artery disease, coronary collateral circulation, serum calprotectin
Correspondence to:

Vahit Demir, MD, Department of Cardiology, Medical Faculty, Bozok University, Çapanoğlu Mah. Cemil Çiçek Cad Bozok Üniversitesi Erdoğan Akdağ Yerleşkesi Atatürk Yolu 7. Km, 66100 Azizli/Yozgat, Turkey, phone: +903542127010, email: dr.vdemir@hotmail.com Received: September 1, 2019 Revision accepted: October 16, 2019. Published online: October 16, 2019. Kardiol Pol. 2019; 77 (12): 1155-1162 doi:10.33963/KP.15023 Copyright by the Author(s), 2019

\section{ABSTRACT}

BACKGROUND In patients with chronic stable coronary artery disease (CAD), well-developed coronary collateral circulation (CCC) is known to reduce long-term mortality.

AIMS The objective of this study was to determine the relationship of serum calprotectin (S100A8/S100A9), angiopoietin-1 (Ang-1) and angiopoietin-2 (Ang-2) concentrations with CCC in patients with stable CAD. METHODS This prospective cross-sectional study included 147 patients with stable angina pectoris. The Cohen-Rentrop classification was used to assess CCC. Patients were divided into 2 groups: with poor CCC (Cohen-Rentrop score, $0-1 ; n=79$ ) and with good CCC (Cohen-Rentrop score, 2-3; $n=68$ ). Serum calprotectin, Ang-1, and Ang-2 concentrations were compated between groups.

RESULTS Compared with the group with good CCC, serum calprotectin and Ang-1 levels were higher $(P<0.01$ and $P<0.001$, respectively), while serum Ang-2 levels were lower $(P<0.01)$ in the poor-CCC group. C-reactive protein levels showed a moderate positive correlation with calprotectin levels $(r=0.359$; $P<0.001)$. In a multivariate regression analysis, only calprotectin $(P<0.05)$ and Ang- $1(P<0.05)$ were found to be independent predictors of good and poor CCC.

CONCLUSIONS Our study showed that Ang-2 levels were lower, while serum calprotectin and Ang-1 levels were higher, in patients with stable CAD and poor CCC regardless of the complexity and severity of coronary arteriosclerosis. If these results are confirmed in future studies, calprotectin may be considered a useful biomarker for guiding anti-ischemic treatment.

INTRODUCTION Coronary artery disease (CAD) is the leading cause of mortality and morbidity worldwide. ${ }^{1}$ In the clinical prognosis of CAD, the complexity and severity of atherosclerosis is as important as the resistance of the heart to ischemia. The myocardial tissue with a sufficiently rich collateral network is more resistant to ischemia. In patients with occluded coronary arteries, the signs and symptoms of CAD may be milder. In patients with chronic stable CAD, well-developed coronary collateral circulation (CCC) reduces long-term mortality. ${ }^{2}$ The development of CCC in response to severe coronary stenosis may vary between individuals. The factors that underlie these differences have not been fully elucidated so far.

Two mechanisms, namely angiogenesis and arteriogenesis, are known be involved in the development of coronary collaterals. Angiogenesis is the process of growth, expansion, and restructuring of primitive vessels to form a complex and mature network..$^{2,3}$ Arteriogenesis 


\section{WHAT'S NEW?}

This is the first study in the literature that investigated the relationship between the serum calprotectin level and coronary collateral circulation (CCC) in patients with stable coronary artery disease (CAD). In our study group, which was homogenous in terms of cardiovascular risk factors, serum calprotectin and angiopoietin-1 levels were high in those with stable $C A D$ and poor $C C C$ regardless of the extent and severity of CAD. Calprotectin, an inflammatory marker, may be therefore an indicator of poor CCC.

is the conversion of pre-existing collateral arterioles into muscular collateral arteries. Angiopoietins are one of the angiogenetic regulators involved in the angiogenic cascade. Angiopoietins modulate vascular development and restructuring during angiogenesis and inflammation. ${ }^{3}$ Angiopoietin-1 (Ang-1) released from the endothelium affects vascular stabilization and maturation to a great extent. By inhibiting the release of adhesion molecules, it demonstrates an anti-inflammatory effect associated with the activity of the vascular endothelial growth factor. ${ }^{4}$ Angiopoietin-2 (Ang-2) released from the Weibel-Palade bodies is a competitive inhibitor of Ang-1, which limits its effect by binding to Tie-2 receptors. Angiopoietin-2 makes the endothelium susceptible to proinflammatory factors and facilitates angiogenesis mediated by the vascular endothelial growth factor. ${ }^{5-7}$

Calprotectin, a member of the $\mathrm{S} 100$ family, is a heterodimeric calcium-binding protein complex with 2 subunits-S100A8 and S100A9. It is a large protein found in leukocytes (monocytes and macrophages) that constitutes up to half of the cytosolic proteins in neutrophil granulocytes. Calprotectin is released by neutrophils and monocytes during the interaction of the inflammation-activated endothelium and monocytes. It binds to endothelial cells and modulates the transendothelial migration of leukocytes. ${ }^{8,9}$ There have been no studies on the role of serum calprotectin in the development of collateral vessels.

For patients who cannot undergo coronary artery revascularization-especially those who do not benefit from a conventional therapy-new therapeutic approaches are needed to achieve CCC formation. For this purpose, we investigated the relationship between serum calprotectin, Ang-1, and Ang-2 levels and CCC in patients with severe stenosis in at least 1 coronary artery.

METHODS Study design and population This prospective cross-sectional study was carried out at the Department of Cardiology, Medical Faculty, Bozok University, Turkey. We enrolled 147 consecutive patients who underwent coronary angiography from June 2016 to January 2019 and who showed stenosis greater than $90 \%$ in at least 1 coronary artery. The exclusion criteria were as follows: a history of coronary revascularization, acute coronary syndrome in the last 6 months, moderate and severe valvular disease, pericardial disease, congenital heart disease, cardiomyopathy, acute infection or chronic inflammatory disease, renal dysfunction (estimated glomerular filtration rate $<50 \mathrm{ml} / \mathrm{min} / 1.73 \mathrm{~m}^{2}$ ), as well as liver dysfunction (transaminase levels 3 times higher than the upper limit of normal). Patients who used antihypertensive, lipidlowering, antiplatelet, and anticoagulant drugs were also excluded. The study protocol was approved by the local ethics committee (decision no., 11/05-04.05.2016). Participants were informed about the aim of the study and provided their written consent before enrollment.

All patients underwent physical examination, and data on their demographic characteristics, cardiovascular risk factors, and concomitant medication were obtained. Transthoracic echocardiography was performed with the Philips Affiniti 50 device (Philips Healthcare, Eindhoven, the Netherlands) according to the recommendations of the American Society of Echocardiography. Left ventricular ejection fraction was calculated using the modified Simpson method. Diabetes was defined as a fasting blood glucose level of $126 \mathrm{mg} / \mathrm{dl}$ or higher or the use of oral antidiabetic medication and insulin. Hypertension was defined as a systolic blood pressure of $140 \mathrm{~mm} \mathrm{Hg}$ or higher, diastolic blood pressure of $90 \mathrm{~mm} \mathrm{Hg}$ or higher, or the use of antihypertensive drugs. Hyperlipidemia was defined as a total cholesterol level of $200 \mathrm{mg} / \mathrm{dl}$ or higher or the use of lipid-lowering drugs. Finally, smoking was defined as current smoking. Body mass index was calculated by dividing body weight by the square of the height $\left(\mathrm{kg} / \mathrm{m}^{2}\right)$.

Laboratory tests In the morning after admission, fasting venous blood samples were collected in 5-ml BD Vacutainer SST II Advance tubes (Becton, Dickinson and Company, Franklin Lakes, New Jersey, United States) and centrifuged at $1300 \times g$ for 10 minutes after completion of clotting. The separated sera were stored at $-80^{\circ} \mathrm{C}$ until measurement. Serum calprotectin, Ang-1, and Ang-2 levels were analyzed using the Human ELISA kits (Sunlong Biotech Co., Ltd., Hangzhou, China). The values of calprotectin, Ang-1, and Ang-2 were expressed in $\mathrm{ng} / \mathrm{ml}$. Additionally, a complete blood count was measured with the XN-1000 analyzer (Sysmex Ameria Inc., Lincolnshire, Illinois, United States), while C-reactive protein (CRP) level, lipid profile, as well as liver and kidney function parameters, with the Architect ci4100 automated analyzer (Abbott, Abbott Park, Illinois, United States).

Coronary angiography and coronary collateral circulation scoring Coronary angiography was performed through the right femoral artery 
using the standard Judkins technique. Coronary arteries were visualized in the cranial and caudal projections, in the right and left oblique views. Iopromide (Ultravist 370, Schering AG, Berlin, Germany) was used as a contrast agent. The angiograms were evaluated by 2 experienced cardiologists who were blinded to patients' clinical characteristics and laboratory values. The interobserver agreement of the Cohen-Rentrop scores was assessed based on a random sample of 30 coronary angiograms $(\mathrm{x}=0.621 ; P<0.001)$. Stenosis and the length of lesions in the coronary arteries were calculated quantitatively using the Digital Imaging and Communications in Medicine program (DICOM, Arlington, Virginia, United States). The CCC was evaluated using the Cohen-Rentrop score and was classified as stage 0 to 3 according to contrast agent concentration in the occluded artery. ${ }^{10}$ The classification is defined as follows: 0 -no significant collateral circulation; 1 -collateral circulation to the lateral branches, not reaching the epicardial artery; 2-partial filling of the epicardial artery; 3-full filling of the epicardial artery. For all patients, the extent and complexity of CAD was evaluated by calculating the SYNTAX score, using a web-based program available at www.syntaxscore.com..$^{11}$ The severity of coronary lesions was also calculated using the Gensini score. ${ }^{12}$ Patients were classified according to the Cohen-Rentrop score into a group with poor CCC (Cohen-Rentrop score, $0-1 ; n=79$ ) and a group with good CCC (Cohen-Rentrop score, $2-3 ; \mathrm{n}=68$ ).

Statistical analysis Statistical analysis was performed using the SPSS software, version 21 (SPSS Inc., Chicago, Illinois, United States). Normal distribution of variables was verified with the Kolmogorov-Smirnov test in a sample of more than 30 and with the Shapiro-Wilk test in a group of less than 30 . The descriptive statistics of continuous variables were expressed as mean (SD) or median (minimum-maximum). Data with normal distribution were evaluated using parametric tests, and those with nonnormal distribution, with nonparametric tests. Categorical variables were examined with the $\mathrm{X}^{2}$ test or Fisher exact test, and continuous variables with the $t$ test or Mann-Whitney test. The KruskalWallis test was used for the comparison of multiple groups. For correlation analysis, the Pearson or Spearman correlation tests were performed according to data distribution. The parameters were evaluated by a univariate logistic regression analysis in order to show poor coronary collateral development. The parameters with a $P$ value of 0.2 or lower in the univariate logistic regression analysis were reevaluated by a multivariate logistic regression analysis. The values were expressed as mean (SD). In all analyses, a $P$ value of less than 0.05 was considered significant.
RESULTS A total of 248 patients were evaluated. Ten patients were excluded from the study due to a history of acute coronary syndrome in the last month; 8 patients, due to a history of coronary artery bypass graft surgery; 13 , due to a history of percutaneous coronary intervention (PCI); 50 , due to noncompliance with the inclusion criteria; 10 , due to moderate-to-severe heart valve disease; 6 , due to renal failure; and 4, due to acute infection and chronic inflammatory disease. This resulted in the final study sample of 147 patients (100 women, 47 men), icluding 79 individuals with poor CCC (mean [SD] age, 62 [9] years) and 68 with good CCC (mean [SD] age, 60 [7] years).

There were no differences between groups in terms of age, sex, body mass index, heart rate, diabetes mellitus, hyperlipidemia, hypertension, and smoking (TABLE1). The levels of fasting blood glucose, creatinine, alanine aminotransferase, aspartate aminotransferase, albumin, total cholesterol, and triglycerides were similar in both groups. White blood cell count, low-density lipoprotein cholesterol levels, lymphocyte count, and CRP levels were higher in patients with poor CCC than in those with good CCC. High-density lipoprotein cholesterol levels were lower in the poor-CCC group than in the good-CCC group. No differences in the echocardiographic data were noted between groups (TABLE2).

There were also no differences between the groups in the results of coronary angiography such as the number of affected vessels, percentage of vessel stenosis, vessels involved, length, location, and type of lesions, as well as the Gensini and SYNTAX scores (TABlE 3).

The median serum calprotectin level in the poor-CCC group was $39.1 \%$ higher than in the good-CCC group. Similarly, the median Ang-1 level in the poor-CCC group was 22\% higher than in the good-CCC group. However, median serum Ang-2 level in the poor-CCC group was $19.9 \%$ lower than in the good-CCC group. Data are presented in TABLE 3 .

The correlation analysis demonstrated that the CRP values were positively correlated with calprotectin levels $(r=0.359 ; P<0.001)$ (FIGURE 1). There were no significant correlations between CRP and Ang-1 or Ang-2 levels.

In the multivariate analysis including age $(\beta=-0.094, P=0.274)$, calprotectin $(\beta=-0.185$, $P<0.05)$, Ang-1 ( $\beta=-0.276, P<0.005)$, Ang-2 $(\beta=0.102, P=0.226)$, the Gensini score $(\beta=0,019$, $P=0.841)$, and the SYNTAX score $(\beta=-0.175$, $P=0.058$ ), only calprotectin and Ang-1 were found to be independent predictors of good or poor CCC $(R=0.426, P<0.001)$.

DISCUSSION The current study showed that patients with poor CCC had lower Ang-2 and higher calprotectin and Ang-1 levels as compared with patients with good CCC. An elevated 
TABLE 1 Demographic, clinical, and laboratory data of the study groups

\begin{tabular}{|c|c|c|c|}
\hline Parameter & $\begin{array}{r}\text { Poor CCC } \\
(n=79)\end{array}$ & $\begin{array}{c}\text { Good CCC } \\
(n=68)\end{array}$ & $P$ value \\
\hline Age, y & $62(9)$ & $60(7)$ & 0.25 \\
\hline Sex, female/male & $55 / 24$ & $45 / 23$ & 0.66 \\
\hline $\mathrm{BMI}, \mathrm{kg} / \mathrm{m}^{2}$ & $24.5(2.0)$ & $24.7(2.5)$ & 0.58 \\
\hline Smoking, n (\%) & $31(39)$ & $30(44)$ & 0.58 \\
\hline Hyperlipidemia, n (\%) & $45(57)$ & $31(46)$ & 0.18 \\
\hline Diabetes mellitus, n (\%) & $39(49)$ & $38(56)$ & 0.45 \\
\hline Hypertension, n (\%) & $35(44)$ & $35(52)$ & 0.41 \\
\hline SBP, mm Hg & $134(7)$ & $133(9)$ & 0.35 \\
\hline $\mathrm{DBP}, \mathrm{mm} \mathrm{Hg}$ & $81(5)$ & $80(5)$ & 0.44 \\
\hline Heart rate, bpm & $79(5)$ & $78(6)$ & 0.13 \\
\hline $\mathrm{FBG}, \mathrm{mg} / \mathrm{dl}$ & $112(25)$ & $117(40)$ & 0.40 \\
\hline Creatinine, mg/dl & $0.87(0.13)$ & $0.85(0.14)$ & 0.26 \\
\hline Total cholesterol, mg/dl & $225(46)$ & $214(41)$ & 0.14 \\
\hline Triglycerides, mg/dl & $192(103)$ & $181(98)$ & 0.55 \\
\hline $\mathrm{HDL}-\mathrm{C}, \mathrm{mg} / \mathrm{dl}$ & $38(10)$ & $43(10)$ & $<0.005$ \\
\hline LDL-C, mg/dl & $139(34)$ & $127(30)$ & $<0.05$ \\
\hline AST, U/I & $18(8)$ & $18(5)$ & 0.84 \\
\hline ALT, U/I & $21(4)$ & $22(5)$ & 0.22 \\
\hline Albumin, g/dl & $4.0(0.3)$ & $4.1(0.2)$ & 0.14 \\
\hline White blood cells, $10^{3} / \mathrm{mm}^{3}$ & $9.1(1.8)$ & $8.2(1.9)$ & $<0.01$ \\
\hline Hemoglobin, g/dl & $14.5(1.0)$ & $14.4(1.3)$ & 0.74 \\
\hline Neutrophils, $10^{3} / \mathrm{mm}^{3}$ & $4.8(1.5)$ & $4.8(1.7)$ & 0.99 \\
\hline Lymphocytes, $10^{3} / \mathrm{mm}^{3}$ & $3.1(1.2)$ & $2.6(1.1)$ & $<0.01$ \\
\hline Platelets, $10^{3} / \mathrm{mm}^{3}$ & $240(44)$ & $247(63)$ & 0.48 \\
\hline MPV, fl & $9.9(0.7)$ & $10.0(0.9)$ & 0.66 \\
\hline CRP, mg/dl & $7.6(4.1)$ & $5.7(3.7)$ & $<0.01$ \\
\hline
\end{tabular}

Data are presented as mean (SD) unless otherwise indicated. $P$ values were compared using the $t$ test or $\chi^{2}$ test, as appropriate. $P<0.05$ was considered significant.

SI conversion factors: to convert C-reactive protein to $\mathrm{nmol} / \mathrm{I}$, multiply by 9.524 ; LDL-C, HDL-C, and total cholesterol to $\mathrm{mmol} / \mathrm{l}$, by 0.0259 ; triglycerides to $\mathrm{mmol} / \mathrm{l}$, by 0.0113 ; hemoglobin to $\mathrm{g} / \mathrm{l}$, by 10 .

Abbreviations: ALT, alanine aminotransferase; AST, aspartate aminotransferase; BMI, body mass index; CCC, coronary collateral circulation; CRP, C-reactive protein; DBP, diastolic blood pressure; FBG, fasting blood glucose; HDL-C, high-density lipoprotein cholesterol; LDL-C, low-density lipoprotein cholesterol; MPV, mean platelet volume; SBP, systolic blood pressure

calprotectin level, which is an inflammatory marker, and a low level of Ang-2, which plays a role in arteriogenesis, suggested that these molecules may be important for CCC.

Current experimental studies show a key role of angiopoietins in angio- and arteriogenesis. ${ }^{4}$ What is more, growth factors released from inflammatory cells (eg, monocytes) that attack the ischemic region in angiogenesis and arteriogenesis are also involved in this process. ${ }^{1,3,13,14}$ Some cytokines initiate an inflammatory response by activating transcription factors. This stimulates the endothelium, resulting in a prompt release of presynthesized and stored molecules such as von Willebrand factor, P-selectin, CD63, interleukin 8, endothelin-1, tissue plasminogen activator, and Ang-2.13 All of them are involved in the control a rapid endothelial response, hemostasis, inflammation, hemodynamic adaptation, and vascular permeability.

Calprotectin is a newly identified cytokine that can be detected in the cytoplasm of healthy individuals. Its concentrations are locally increased in tissue trauma and inflammation. 
TABLE 2 Results of transthoracic echocardiography in the study groups

\begin{tabular}{llll} 
Parameter & $\begin{array}{c}\text { Poor CCC } \\
(\mathrm{n}=79)\end{array}$ & $\begin{array}{c}\text { Good CCC } \\
(\mathrm{n}=68)\end{array}$ & P value \\
\hline LVEF, \% & $53.7(3.7)$ & $52.6(3.5)$ & 0.08 \\
\hline Aortic diameter, cm & $2.31(0.27)$ & $2.33(0.23)$ & 0.67 \\
\hline Left atrial diameter, cm & $3.52(0.37)$ & $3.55(0.41)$ & 0.74 \\
\hline LVEDD, cm & $4.56(0.33)$ & $4.61(0.33)$ & 0.39 \\
\hline LVESD, cm & $2.88(0.64)$ & $2.75(0.54)$ & 0.26 \\
\hline IVSd, cm & $1.10(0.10)$ & $1.13(0.13)$ & 0.17 \\
\hline PWd, cm & $1.00(0.06)$ & $0.99(0.11)$ & 0.45 \\
\hline Mitral E-wave velocity, cm/s & $62(19)$ & $55(20)$ & 0.12 \\
\hline Mitral E/A ratio & $0.84(0.35)$ & $0.74(0.39)$ & 0.18 \\
\hline
\end{tabular}

Data are presented as mean (SD). $P<0.05$ was considered significant.

Abbreviation: IVSd, interventricular septal thickness; LVEDD, left ventricular end-diastolic diameter; LVEF, left ventricular ejection fraction; LVESD, left ventricular end-systolic diameter; PWd, posterior wall thickness at end-diastole, others, see TABLE 1

TABLE 3 Results of coronary angiography and serum calprotectin, angiopoietin-1, and angiopoietin-2 levels in the study groups

\begin{tabular}{|c|c|c|c|c|}
\hline \multicolumn{2}{|l|}{ Parameter } & $\begin{array}{r}\text { Poor CCC } \\
(n=79)\end{array}$ & $\begin{array}{c}\text { Good CCC } \\
(n=68)\end{array}$ & Pvalue \\
\hline \multicolumn{2}{|l|}{ Affected vessels, $n$} & $2.2(0.75)$ & $2.3(0.67)$ & 0.27 \\
\hline \multirow[t]{3}{*}{ Vessel involved } & LAD & $12(15)$ & $8(12)$ & \multirow[t]{3}{*}{0.74} \\
\hline & $\mathrm{LCx}$ & $33(42)$ & $27(40)$ & \\
\hline & $\mathrm{RCA}$ & $34(43)$ & $33(48)$ & \\
\hline \multicolumn{2}{|l|}{ Lesion length, mm } & $24.4(7.3)$ & $25.8(6.3)$ & 0.25 \\
\hline \multirow[t]{3}{*}{ Lesion location } & Proximal & $34(43)$ & $33(48)$ & \multirow[t]{3}{*}{0.53} \\
\hline & Medial & $32(41)$ & $28(41)$ & \\
\hline & Distal & $13(16)$ & $7(11)$ & \\
\hline \multicolumn{2}{|l|}{ Vessel stenosis, \% } & $98.5(2.6)$ & $98.6(2.6)$ & 0.78 \\
\hline \multirow[t]{3}{*}{ Lesion type } & $A$ & $24(30)$ & $22(32)$ & \multirow[t]{3}{*}{0.69} \\
\hline & $B$ & $26(33)$ & $18(27)$ & \\
\hline & $\mathrm{C}$ & $29(37)$ & $28(41)$ & \\
\hline \multirow[t]{4}{*}{ Rentrop class, n (\%) } & 0 & $31(39)$ & - & \multirow[t]{4}{*}{$<0.001$} \\
\hline & 1 & $48(61)$ & - & \\
\hline & 2 & - & $22(32)$ & \\
\hline & 3 & - & $46(68)$ & \\
\hline \multicolumn{2}{|l|}{ Gensini score } & $36(19)$ & $34(16)$ & 0.50 \\
\hline \multicolumn{2}{|l|}{ SYNTAX score } & $23(9)$ & $21(8)$ & 0.18 \\
\hline \multicolumn{2}{|c|}{ Calprotectin, ng/ml, median (range) } & $718(465-1275)$ & $516(352-794)$ & $<0.01$ \\
\hline \multicolumn{2}{|c|}{ Angiopoietin 1, ng/ml, median (range) } & $2.11(1.83-2.71)$ & $1.73(1.35-2.08)$ & $<0.001$ \\
\hline \multicolumn{2}{|c|}{ Angiopoietin 2, ng/ml, median (range) } & $6.88(3.90-11.03)$ & $8.59(6.57-14.60)$ & $<0.01$ \\
\hline
\end{tabular}

Data are presented as mean (SD) unless otherwise indicated. $P<0.05$ was considered significant.

Abbreviations: LAD, left anterior descending artery; LCx, left circumflex artery; RCA, right coronary artery, others, see TABLE 1 


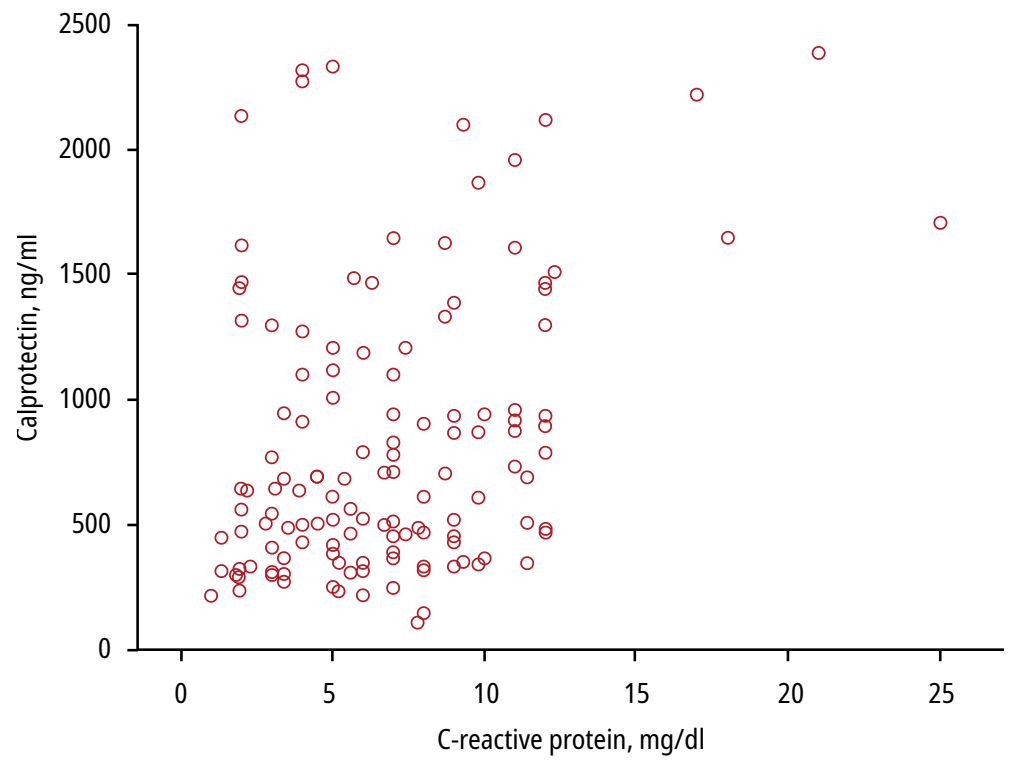

FIGURE 1 Correlation between serum calprotectin and C-reactive protein levels

Calprotectin, released from activated granulocytes and macrophages during inflammation, has a proinflammatory effect. ${ }^{5,15}$ Compared with healthy individuals, serum calprotectin levels were found to be increased in patients with CAD. In addition, this increase is associated with cardiovascular mortality. ${ }^{15,16}$ Moreover, increased all-cause mortality was reported in patients without CAD but with high serum calprotectin levels. ${ }^{17}$ Similarly, in our study, the levels of CRP, which is a marker of inflammation, were higher in the poor-CCC group. Moreover, they showed a positive correlation with calprotectin levels. Although inflammation is a basic defense mechanism of the human body, persistently elevated values of some inflammatory markers considerably affect the development and prognosis of cardiovascular diseases. ${ }^{18}$ It was reported that calprotectin catalyzes the formation of reactive oxygen species by activating NADPH oxidase, as well as is involved in the pathogenesis of atherosclerosis by interacting with the receptor for advanced glycation end products and the toll-like receptor and by accelerating the signaling pathway. ${ }^{13}$ Katashima et al ${ }^{19}$ reported high serum calprotectin levels in patients with acute myocardial infarction. Additionally, the peak calprotectin levels were positively related to peak white blood cell and neutrophil counts along with peak creatine kinase MB isoenzyme and CRP levels. In a study by Jensen et $a l,{ }^{20}$ in 41 patients with ST-segment elevation myocardial infarction successfully treated with PCI, calprotectin levels were higher in 13 patients who died after a mean follow-up of 12 months as compared with survivors $(0.209 \mathrm{mg} / \mathrm{l}$ vs $0.174 \mathrm{mg} / \mathrm{l}$, respectively; $P<0.001)$. Calprotectin was associated with an adverse clinical outcome in patients with suspected chest pain. In another study, including 871 consecutive patients with suspected acute coronary syndrome and with a long-term follow-up of approximately 84 months, the calprotectin level was associated with all-cause mortality (hazard ratio, 1.94; 95\% CI, 1.42-2.66; $P<0.001$ ) and recurrent acute myocardial infarction (hazard ratio, 1.60; 95\% CI, 1.06-2.41; $P=0.024){ }^{21}$ These findings emphasize the prognostic significance of calprotectin. ${ }^{20,21}$ Since patients with acute coronary syndrome were excluded from our study, no comparison was possible; however, high serum calprotectin levels were remarkable in patients with poor CCC.

Several studies have shown that inflammatory process and growth factors play an important role in the development of CCC in myocardial ischemia. ${ }^{13,22-26}$ Some biomarkers may be useful for demonstrating the diversity of CCC development. As previously mentioned, calprotectin, Ang-1, and Ang-2 are biomarkers involved in angiogenesis and inflammation. The calprotectin level increases in inflammatory events, and inflammation considerably affects the onset and progression of atherosclerosis. Angiopoietin- 1 and -2 are involved in vessel maturation and angiogenesis. In our study, calprotectin and Ang-1 levels were lower and Ang-2 levels were higher in patients with well-developed CCC. All these findings suggest more intensive angiogenesis in the heart with good collateral circulation.

In the literature, a few studies found a relationship between elevated plasma Ang-1 levels and CCC development, as well as showed that patients with high plasma Ang-1 and low plasma Ang-2 levels have less developed CCC. ${ }^{4}$ In a case-control study that included 695 patients with acute coronary syndrome and 690 healthy individuals, Ang-2 served as a biomarker of acute myocardial infarction..$^{24}$ In our study, calprotectin and Ang-1 levels were low and Ang-2 levels were high in patients with good CCC, which is consistent with literature data. Furthermore, in the multivariate analysis, calprotectin and Ang-1 were independently associated with poor CCC. Even more importantly, this relationship was demonstrated in patients with stable CAD, while it is known that calprotectin concentrations are higher in patients with acute coronary syndrome. ${ }^{20,21}$ It is suggested that Ang- 1 can prevent remodeling by providing vascular integrity and correct impaired balance by shifting the ratio of Ang-1 to Ang-2 towards Ang-1. Furthermore, high levels of calprotectin in patients without acute coronary syndrome suggest that subclinical inflammation in stable CAD may have a negative effect on CCC.

In a study by Gok et al, ${ }^{27}$ higher CRP levels positively correlated with poor CCC and lower levels of ischemia-modified albumin in patients with stable CAD. Our findings also confirmed that the CRP level was higher in the poor-CCC group and, additionally, CRP was significantly correlated with serum calprotectin levels. Most likely, 
this was related to the high inflammatory status of patients with poor CCC. The study by Sen et $\mathrm{al}^{28}$ also underlined the relation between the CRP value and poor development of CCC. It was reported that higher CRP levels were associated with a higher rate of adverse clinical events and higher probability of non-infarct-related chronic total occlusion in patients with acute ST-segment elevation myocardial infarction. All these findings may reflect the continuous process of CCC development.

Although cardiovascular risk factors have been shown to have negative effects on the development of CCC, ${ }^{5}$ there was no significant difference between our study groups in terms of classic cardiovascular risk factors other than high-density and low-density lipoprotein cholesterol. This may be due to the low number of patients and the exclusion of those who used medications known to reduce cardiovascular risk.

Complex long-term mechanisms may be involved in the interplay between CCC and serum calprotectin and angiopoietins. The elucidation of the relationship between these markers and CCC could help diagnose high-risk patients earlier and initiate aggressive treatment. In addition, if these mechanisms are clarified, new treatment strategies can be developed for high-risk patients with CAD.

Limitations The most important limitation of our study is the small number of patients. This might have prevented the identification of additional risk factors that may affect the development of CCC. Another limitation is the use of coronary angiography to detect CCC. Collateral vessels with a diameter of at least $100 \mu \mathrm{m}$ can be angiographically visualized, but not the smaller ones. Therefore, patients with suspected inadequate CCC network may have collateral vessels that cannot be detected using this method. Furthermore, we excluded patients on antihypertensive, lipid-lowering, antiplatelet, and anticoagulant drugs, which may affect the development of CCC. This can be a limitation, since it might not reflect a real-life setting. Thus, the results may not be applied to the general population.

Conclusion In our study, we compared 2 patient groups with similar cardiac and metabolic characteristics and no difference in the severity and complexity of risk factors for CAD and atherosclerosis. As a result, calprotectin and Ang-1 were independently associated with poor CCC. Considering the relationship between the serum calprotectin level and poor prognosis in patients with $C A D$, poor $C C C$ associated with the serum calprotectin concentration may be one of the causes of this poor prognosis. Understanding the role of calprotectin and Ang-1 may lead to the development of new treatment strategies for patients who cannot benefit from conventional therapies such as coronary bypass surgery or PCI. What is more, both proteins can serve as biomarkers of poor CCC. Therefore, more comprehensive multicenter prospective studies should be performed to further elucidate the relationship between serum calprotectin and angiopoietins and the development of CCC.

\section{ARTICLE INFORMATION}

ACKNOWLEDGMENTS This study was supported by the Directorate of Scientific Research Projects, Bozok University (project decision no., 6602a-TF/16-40; to VD). CONFLICT OF INTEREST None declared.

OPEN ACCESS This is an Open Access article distributed under the terms of the Creative Commons Attribution-NonCommercial-NoDerivatives 4.0 International License (CC BY-NC-ND 4.0), allowing third parties to download articles and share them with others, provided the original work is properly cited, not changed in any way, distributed under the same license, and used for noncommercial purposes only. For commercial use, please contact the journal office at kardiologiapolska@ptkardio.pl.

HOW TO CITE Demir V, Ede H, Ercan M, et al. Relationship of serum calprotectin, angiopoietin-1, and angiopoietin-2 levels with coronary collateral circulation in patients with stable coronary artery disease. Kardiol Pol. 2019; 77: 1155-1162. doi:10.33963/KP.15023

\section{REFERENCES}

1 Zehir R, Güner A, Hayiroglu MI, et al. Clinical usefulness of epicardial adipose tissue in patients with high-intermediate pre-test probability for coronary artery disease. Kardiol Pol. 2018; 76: 1002-1008.

2 Kersten JR, Pagel PS, Chilian WM, Warltier DC. Multifactorial basis for coronary collateralization: a complex adaptive response to ischemia. Cardiovasc Res. 1999; 43: 44-57.

3 Seiler C. The human coronary collateral circulation. Eur J Clin Invest. 2010; 40: 465-476.

4 Bowler E, Oltean S. Alternative splicing in angiogenesis. IntJ Mol Sci. 2019; 20: E2067. 5 Tron C, Donohue TJ, Bach RG, et al. Differential characterization of human coronary collateral blood flow velocity. Am Heart J. 1996; 132: 508-515.

6 Mitsuma W, Kodama M, Hirono S, et al. Angiopoietin-1, angiopoietin-2 and tie-2 in the coronary circulation of patients with and without coronary collateral vessels. Circ J. 2007; 71: 343-347.

7 Rockstroh J, Brown BG. Coronary collateral size, flow capacity, and growth: estimates from the angiogram in patients with obstructive coronary disease. Circulation. 2002; 105: 168-173.

8 Shabani F, Farasat A, Mahdavi M, Gheibi N. Calprotectin (S100A8/S100A9): a key protein between inflammation and cancer. Inflamm Res. 2018; 67: 801-812.

9 Abildtrup M, Kingsley GH, Scott DL. Calprotectin as a biomarker for rheumatoid arthritis: a systematic review. J Rheumatol. 2015; 42: 760-770.

10 Rentrop KP, Cohen M, Blanke H, Phillips RA. Changes in collateral channel filling immediately after controlled coro-nary artery occlusion by an angioplasty balloon in human subjects. J Am Coll Cardiol. 1985; 5: 587-592.

11 Athappan G, Patvardhan E, Tuzcu ME, et al. Left main coronary artery stenosis: a meta-analysis of drug-eluting stents versus coronary artery bypass grafting. JACC Cardiovasc Interv. 2013; 6: 1219-1230.

12 Gensini GG. A more meaningful scoring system for determining the severity of coronary heart disease. Am J Cardiol. 1983; 51: 606.

13 Jamaiyar A, Juguilon C, Dong $F$, et al. Cardioprotection during ischemia by coronary collateral growth. Am J Physiol Heart Circ Physiol. 2019; 316: H1-H9.

14 Seiler C, Stoller M, Pitt B, Meier P. The human coronary collateral circulation: development and clinical importance. Eur Heart J. 2013; 34: 2674-2682.

15 Leclerc E, Fritz G, Vetter S.W, Heizmann CW. Binding of $S 100$ proteins to RAGE: an update. Biochim Biophys Acta. 2009; 1793: 993-1007.

16 Healy AM, Pickard MD, Pradhan AD, et al. Platelet expression profiling and clinical validation of myeloid-related protein-14 as a novel determinant of cardiovascular events. Circulation. 2006; 113: 2278-2284.

17 Montagnana M, Danese E, Lippi G. Calprotectin and cardiovascular events. a narrative review. Clin Biochem. 2014; 47: 996-1001.

18 Kosmas CE, Silverio D, Sourlas A, et al. Anti-inflammatory therapy for cardiovascular disease. Ann Transl Med. 2019; 7: 147.

19 Katashima T, Naruko T, Terasaki F, et al. Enhanced Expression of the S100A8/ A9 Complex in Acute Myocardial Infarction Patients. Circ J. 2010; 74: 741-748.

20 Jensen LJ, Pedersen S, Bjerre M, et al. Plasma calprotectin predicts mortality in patients with ST segment elevation myocardial infarction treated with primary percutaneous coronary intervention. J Interv Cardiol. 2010; 23: 123-129.

21 Mjelva OR, Brügger-Andersen T, Pönitz V, et al. Long term prognostic utility of PAPP-A and calprotectin in suspected acute coronary syndrome. Scand Cardiovasc J. 2013; 47: 88-97. 
22 Cui $K$, Lyu S, Song $X$, et al. Effect of coronary collaterals on prognosis in patients undergoing primary percutaneous coronary intervention for acute ST-segment elevation myocardial infarction: a meta-analysis. Angiology. 2018; 69 : 803-811.

23 Joseph G, Soler A, Hutcheson R, et al. Elevated 20-HETE impairs coronary collateral growth in metabolic syndrome via endothelial dysfunction. Am J Physiol Heart Circ Physiol. 2017; 312: H528-H540.

24 Iribarren C, Phelps BH, Darbinian JA, et al. Circulating angiopoietins-1 and -2 , angiopoietin receptor Tie-2 and vascular endothelial growth factor-A as biomarkers of acute myocardial infarction: a prospective nested case-control study. BMC Cardiovasc Disord. 2011; 11: 31.

25 Tan X, Yan K, Ren M, et al. Angiopoietin-2 impairs collateral artery growth associated with the suppression of the infiltration of macrophages in mouse hindlimb ischaemia. J Transl Med. 2016; 14: 306.

26 Akboga MK, Yalcin R, Sahinarslan A, et al. Effect of serum YKL-40 on coronary collateral development and SYNTAX score in stable coronary artery disease. Int J Cardiol. 2016; 224: 323-327.

27 Gök M, Kundi H, Kızltunç $E$, et al. The relationship between ischaemia-modified albumin and good coronary collateral circulation. Kardiol Pol. 2018; 76: 370-375.

28 Şen 0̈, Allahverdiyev S, Topuz M, et al. Clinical significance and determinants of prompt recruitment collaterals during primary percutaneous coronary intervention. Kardiol Pol. 2017; 75: 763-769. 